\title{
A Four-Year Observational Study regarding the Characteristics and Causes of the Ischemic Stroke in Young Adults: one center study report
}

\author{
MUTU Cătălin Cosmin ${ }^{1,2}$, GHINCEA Marius-Ionuț ${ }^{1}$, SUCIU Corina ${ }^{3}$, SUCIU Vlad ${ }^{4}$
}

Corresponding author: MUTU Cătălin Cosmin, E-mail: cosmin.mutu@,ulbsibiu.ro

1. “Lucian Blaga” University of Sibiu, Sibiu, Romania 2. Clinical County Emergency Hospital, CVASIC Research Center, Sibiu, Romania

3. Emergency County Hospital Cluj-Napoca, Romania

4. Private Practice, Neurology, Cluj-Napoca, Romania

\begin{abstract}
Introduction. Stroke is the second cause of mortality worldwide, after the ischemic coronary disease, and the first cause of long term-disability. Recent studies report that ischemic stroke (IS) in young patients is increasing in incidence, even though the overall stroke hospitalization rate is decreasing. Stroke in the young, working individuals significantly affects the quality of life (QoL) and also determines an important, negative economic impact by reducing the workforce. Material and method. The aim of this paper is to present the results of a 4-year observational, retrospective study on all patients between 18-49 years, who suffered from acute IS and were admitted and investigated in the Neurology Clinic of Sibiu and the CVASIC Research Center. Clinical evaluations, imaging investigations, and risk factor evaluations were performed on all patients in order to elucidate the etiology and organize according to the TOAST classification. Results and discussions. 114 patients with ischemic stroke were included in the study. $76 \%$ of the subjects fall into the 40-49 age class. In respect to the TOAST classification, most cases of IS were of UE (rural<urban), while the fewest cases of IS were represented by SAA in both populations. The most frequent vascular risk factors were hypertension, dyslipidemia, smoking and thrombophilia. Confirmed cervical arteries dissection caused $2 / 3$ of the severe carotid stenosis and $45,5 \%$ of the carotid occlusions. Conclusions. Hypertension, dyslipidemia and smoking are the main risk factors for stroke in young patients. The most common TOAST stroke subtype was stroke of undetermined etiology, followed by stroke of other determined etiology (carotid artery dissection and thrombophilia).
\end{abstract}

Keywords: ischemic stroke, young patients, vascular risk factors, TOAST classification, disability,

\section{Introduction}

Stroke is considered to be the second cause of mortality in the world, after the ischemic coronary disease $(1,2)$. Additionally, it is the main cause of long-term disability. Recent studies report that stroke in young patients $(<50$ years) is increasing in incidence, even though the overall stroke hospitalization rate is decreasing. Stroke in the young, working individuals significantly affects the quality of life (QoL) and also determines an important, negative economic impact by reducing the workforce (2). The prevalence of risk factors in young patients differs from those in elderly patients. The most frequent modifiable risk factors for ischemic stroke in young adults are hypertension, dyslipidemia and smoking $(1,2,3)$. According to the TOAST classification, patients with ischemic stroke fall into 5 subtypes: large artery atherosclerosis (LAA), small vessel occlusion (SVO), cardio-embolic stroke (CE), stroke of other determined etiology (OE) and stroke of undetermined etiology (UE). Based on these criteria, the most common stroke subtypes in young patients are $\mathrm{OE}$ and $\operatorname{UE}(3,4)$.

\section{Purpose}

The aim of this paper is to present the results of a 4-year observational, retrospective study on all patients between 18-49 years, who suffered from acute IS and were admitted and investigated in the Neurology Clinic of Sibiu and the CVASIC Research Center. Clinical evaluations, imaging investigations, and risk factor evaluations were performed on all patients in order to elucidate the etiology and organize according to the TOAST classification.

\section{Materials and methods}

We conducted an observational, analytical, retrospective cohort study, that included 114 patients, younger than 50 years of age, who have been admitted to the Neurology Department Sibiu starting from January 2016 until December 2019. The subjects were selected from both The Department of Neurology and The Center for invasive and noninvasive research regarding cardiac and vascular pathology in adults (CVASIC Research Center) databases.

Patients were eligible for inclusion in the study if they were younger than 50 years at the time of disease onset, admitted in the Department of Neurology during the specified period of time and if they had a clinical presentation suggestive for stroke (confirmed to be an ischemic stroke later, by imaging investigations).

\section{Data management}

The risk factors that were taken into consideration were: smoking, alcohol consumption, diabetes, hypertension, high cholesterol, atrial fibrillation, obesity, history of ischemic stroke and myocardial infarction.

The patients were divided into 3 age groups accordingly: 20-29, 30-39 and 40-49 years old.

104 out of a total of 114 patients have been evaluated using carotid Doppler ultrasonography, thereby revealing the presence of carotid artery stenosis (CAS). The degree 
of CAS was classified as follows: 0 - no stenosis; 1 (mild stenosis) - 20-50\%; 2 (moderate stenosis) $-50-70 \% ; 3$ (severe stenosis) $-70-99 \%$ and grade 4 - total vascular occlusion. Furthermore, in respect to the TOAST classification, the IS was classified into 5 subtypes: LAA - stroke due to a large artery atherosclerosis, SAA stroke due to small vessels occlusion, CE - cardioembolic stroke, $\mathrm{OE}$ - stroke of other determined etiology and UE - stroke of undetermined etiology.

\section{Statistical analysis}

We used the Chi-Square statistical test to analyze the risk factors that contribute to the distribution of patients into TOAST subtypes of stroke. We used the Pearson correlation to quantify the distribution of the CAS according to the TOAST classification. Also, a multinomial logistic regression was used in order to identify the risk factors implicated in the development of the CAS. For all these tests, the level of the statistical significance was set to a $\mathrm{p}$-value $<0,05$.

\section{Results}

We evaluated 72 male and 42 female patients with a mean age of 43 years. The patients were divided into 3 age groups accordingly: 20-29, 30-39 and 40-49 years old, the last group being $76,3 \%$ of all cases. $63,1 \%$ of the subjects originate from the rural environment.

In the studied cohort, the most frequently recognized risk factors for ischemic stroke where hypertension, high cholesterol levels and smoking. (figure 1)

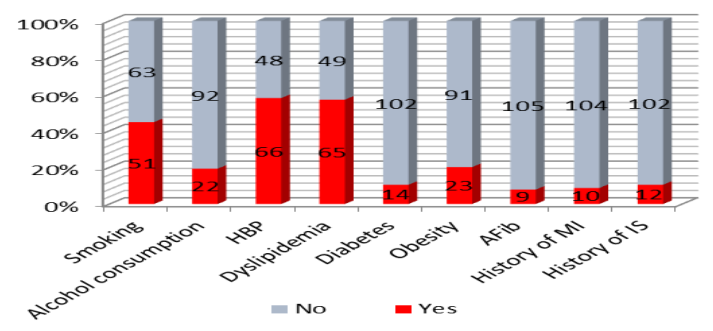

FIG. 1. The distribution of risk factors in the study group (HBP - High blood pressure, AFib - Atrial fibrillation, MI - Myocardial infaction, IS - Ischemic stroke)

In the age group 40-49 years, were found all the patients with atrial fibrillation and $80 \%$ of those with a history of myocardial infarction and ischemic stroke. Men were found with atrial fibrillation more frequently than women (8:1 male to female ratio).

With respect to the TOAST classification, 36,84\% patients had UE, $24,57 \%$ had OE, $16,66 \%$ had $\mathrm{CE}$, $15,79 \%$ had LAA and $6,14 \%$ had SVO. (figure 2)

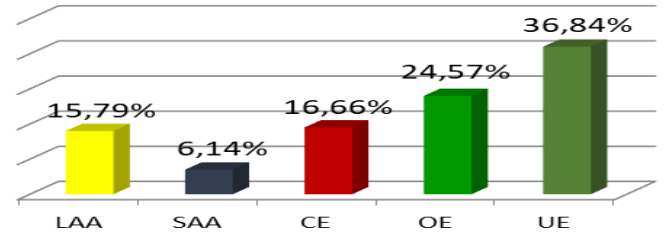

FIG. 2. The percentage distribution of patients based on the TOAST classification
The majority of patients are between $40-49$ years. Men had higher incidence of suffering from stroke in all subtypes, except in UE, where gender distribution was similar.

TABLE 1. The distribution of risk factors within TOAST subtypes of stroke

\begin{tabular}{|l|l|l|l|l|l|}
\hline \multirow{2}{*}{ TOAST Subtypes of Stroke } & LAA & SVO & CE & OE & UE \\
\cline { 2 - 6 } & $\%$ & $\%$ & $\%$ & $\%$ & $\%$ \\
\hline Smoking & 19,6 & 11,8 & 19,6 & 21,5 & 27,5 \\
\hline Alcohol consumption & 18,2 & 18,2 & 18,2 & 18,2 & 27,2 \\
\hline High blood pressure & 27,3 & 7,6 & 18,2 & 16,6 & 30,3 \\
\hline High cholesterol & 20 & 9,2 & 15,4 & 23,1 & 32,3 \\
\hline Diabetes mellitus & 0 & 0 & 21,4 & 35,7 & 42,9 \\
\hline Obesity & 8,7 & 8,7 & 26,1 & 21,7 & 34,8 \\
\hline Atrial fibrillation & 0 & 0 & 100 & 0 & 0 \\
\hline History of MI & 10 & 10 & 50 & 10 & 20 \\
\hline History of ischemic stroke & 8,3 & 8,3 & 16,7 & 25 & 41,7 \\
\hline
\end{tabular}

In UE stroke type, smoking (27,5\%) and alcohol consumption $(27,2 \%)$ predominated slightly when compared to other stroke types. High blood pressure was more predominant in LAA $(27,3 \%)$ and UE $(30,3 \%)$ types. The metabolic risk factors such as high cholesterol, diabetes mellitus and obesity were more frequent in UE subtype, respectively $32,3 \%, 42,9 \%$ and $34,8 \%$ (in text order).Atrial fibrillation and the history of MI were the primary risk factors for CE stroke. However, only 9 out of 19 patients with cardio-embolic stroke had been diagnosed with atrial fibrillation prior to the ischemic stroke. The other causes of cerebral embolism in the study group were: left ventricular and inter-ventricular septal aneurysm, infective endocarditis, mechanic valve prosthesis and mitral valve prolapse. The Chi-Square test revealed the risk factors which contributed to the distribution of patients into the TOAST classification. High blood pressure ( $\mathrm{p}$-value $=0,001)$, history of MI ( $\mathrm{p}$ value $=0,048)$ and AFib ( $p$-value $<0,001)$ have obtained statistically significant values.

TABLE 2. The distribution of patients based on the CAS grading and TOAST subtypes of stroke

\begin{tabular}{|l|l|l|l|l|l|}
\hline & $\begin{array}{l}\text { Without } \\
\text { stenosis }\end{array}$ & $\begin{array}{l}\mathbf{2 0 - 5 0 \%} \\
\text { stenosis }\end{array}$ & $\begin{array}{l}\mathbf{5 0 - 7 0 \%} \\
\text { stenosis }\end{array}$ & $\begin{array}{l}\mathbf{7 0 - 9 9 \%} \\
\text { stenosis }\end{array}$ & $\begin{array}{l}\text { Carotid } \\
\text { occlusion }\end{array}$ \\
\cline { 2 - 6 } & $\%$ & $\%$ & $\%$ & $\%$ & $\%$ \\
\hline LAA & 2,98 & 20 & 60 & 33 & 27,27 \\
\hline SVO & 8,95 & 0 & 0 & 0 & 0 \\
\hline CE & 14,93 & 40 & 0 & 0 & 27,27 \\
\hline OE & 19,40 & 20 & 40 & 66 & 45,46 \\
\hline UE & 53,73 & 20 & 0 & 0 & 0 \\
\hline
\end{tabular}

According to the general guidelines, a CAS of $>50 \%$ was considered to be significant. Patients with LAA had predominantly $(60 \%)$ second grade stenosis, while patients with OE subtype (including carotid dissection) had more frequently third grade stenosis $(66 \%)$ and total carotid occlusion $(45,46 \%)$. Among the studied risk factors, high blood pressure was the risk factor that 
contributed the most in the development of the carotid artery stenosis.

\section{Discussions}

According to medical literature, the most common modifiable risk factors for ischemic stroke in young patients are high blood pressure, dyslipidemia and smoking $(2,5)$. Our study confirms the information from the medical literature.Stroke survivors, even younger individuals, are at high risk of stroke reoccurrence. This can be prevented by correcting the modifiable risk factors and by adopting a healthy lifestyle (smoke and alcohol cessation, low fat and salt diet). (3, 6, 7)

In the present study, UE and OE subtypes were the most frequent. According to the medical literature, UE (24$40 \%)$, OE (9-29\%) and CE (10-34\%) are the most frequent subtypes in young patients $(8,9)$.

However, the data regarding the stroke subtypes in the general population differ in the medical literature from our study (LAA: 9,3-35,8\%, SVO: 15,3-43\%, CE: 827,8\%, OE: $1-3,4 \%$ și UE: $13-28,7 \%)(9,10,11)$.

In this study, OE $(24,57 \%)$ is much higher than in the general population, probably because of the high incidence of carotid artery dissection and thrombophilia in young patients. Also, the small vessel occlusion $(6,14 \%)$ had the most decreased incidence in our study, probably because of the lack of lacunar stroke in young patients (12).In accordance with the medical literature, the carotid artery dissection is an increasingly frequent cause of ischemic stroke in young patients (13). Our study shows that the carotid artery dissection is the main cause for both high grade stenosis and total carotid occlusion. In our study, atrial fibrillation was the primary risk factor for $\mathrm{CE}$ stroke and only almost half of the patients with cardio-embolic stroke had been diagnosed with atrial fibrillation prior to the ischemic stroke (14).

\section{Conclusions}

The most common risk factors for stroke in young patients were hypertension, dyslipidemia and smoking, followed by obesity, alcohol consumption, diabetes and atrial fibrillation. The main subtype of stroke according to the TOAST classification in young adults was stroke of undetermined etiology, followed by stroke of other determined etiology (carotid artery dissection and thrombophilia) and cardioembolic stroke.

Arterial hypertension, atrial fibrillation and history of myocardial infarction have reached statistically significant values in accordance with their contribution to the classification stroke.

The carotid artery dissection is an important cause of ischemic stroke in young patients, causing the majority of severe stenosis and total carotid occlusion.
High blood pressure represents the vascular risk factor that contributes the most to the development of carotid artery stenosis.

Declaration of conflict of interests/Conflict of Interest Statement - The authors declare no conflicting interests regarding this paper at the time of submission.

Informed consent - Informed consent was obtained from all patients included in this study

\section{References:}

1. Palomeras Soler E, Casado Ruiz V. Epidemiology and Risk Factors of Cerebral Ischemia and Ischemic Heart Diseases: Similarities and Differences. CurrCardiol Rev. 2010;6(3):138-49. DOI: $10.2174 / 157340310791658785$

2. Stack CA, Cole JW. Ischemic stroke in young adults. CurrOpinCardiol. DOI: 10.1097/HCO.0000000000000564

3. van Alebeek ME, Arntz RM, Ekker MS, Synhaeve NE, Maaijwee NAMM, Schoonderwaldt H, et al. Risk factors and mechanisms of stroke in young adults: The FUTURE study. J Cereb Blood Flow Metab. 2018;38(9):1631-41. DOI: 10.1177/0271678X17707138

4. Patel AR, Patel AR, Desai S. The Underlying Stroke Etiology: A Comparison of Two Classifications in a Rural Setup. Cureus. 2019;179(7). DOI: 10.7759/cureus.5157

5. Boot E, Ekker MS, Putaala J, Kittner S, De Leeuw FE, Tuladhar AM. Ischaemic stroke in young adults: A global perspective. J Neurol Neurosurg Psychiatry. 2020;91(4):411-7. DOI: 10.1136/jnnp-2019-322424

6. Singhal AB, Biller J, Elkind MS, Fullerton HJ, Jauch EC, Kittner $\mathrm{SJ}$, et al. Recognition and management of stroke in young adults and adolescents. Neurology. 2013;81(12):1089-97. DOI: 10.1161/STROKEAHA.119.024156

7. Dura S. Alcohol and vulnerable youth in Romania. Acta Medica Transilvanica. 2018;23(2):30-32. http://www.amtsibiu.ro/Arhiva/2018/Nr2-enn/Dura.pdf.

8. Smajlović D. Strokes in young adults: Epidemiology and prevention. Vasc Health Risk Manag. 2015;11:157-64. DOI: 10.2147/VHRM.S53203

9. Bejot Y, Caillier M, Ben Salem D, Couvreur G, Rouaud O, Osseby G V., et al. Ischaemic stroke subtypes and associated risk factors: A French population based study. J Neurol Neurosurg Psychiatry. 2008;79(12):1344-8. DOI: 10.1136/jnnp.2008.150318

10. Hajat C, Heuschmann PU, Coshall C, Padayachee S, Chambers J, Rudd AG, et al. Incidence of aetiological subtypes of stroke in a multi-ethnic population based study: The south London Stroke Register. J Neurol Neurosurg Psychiatry. 2011;82(5):527-33. https://doi.org/10.1136/jnnp.2010.222919

11. Mutu C, Minea N, Pereanu M. Incidence of ischemic stroke subtypes among the population of the county of Sibiu: a prospective study. Acta Medica Transilvanica 2012;17(3):29-31. http://www.amtsibiu.ro/Arhiva/2012/Nr3-en/Mutu.pdf

12. Arboix A, Estevez S, Rouco R, Oliveres M, García-Eroles L, Massons J. Clinical characteristics of acute lacunar stroke in young adults. Expert Rev Neurother. 2015;15(7):825-31. DOI: $10.1586 / 14737175.2015 .1049997$

13. Rao AS, Makaroun MS, Marone LK, Cho JS, Rhee R, Chaer RA. Long-term outcomes of internal carotid artery dissection. J Vasc Surg. 2011;54(2):370-5. DOI: 10.1016/j.jvs.2011.02.059

14. Orga-Dumitriu D, Teodoru D, Tanasescu C, Teodoru A. Actualities in thromboembolic risk management in the patients with atrial fibrillation. Acta Medica Transilvanica 2012;17(3):103104. http://www.amtsibiu.ro/Arhiva/2012/Nr3-en/Orga.pdf 